\title{
Conductance Switching in Molecular Self- Assembled Monolayers for Application of Data Storage
}

Chao Chen ${ }^{\dagger}$, Sha Yang ${ }^{\dagger}$, Guirong Sü, Jingtai $\mathrm{Li}^{\dagger}$, Ji-Chang Ren ${ }^{\dagger}$, and Wei Liu ${ }^{\dagger, *}$

${ }^{\dagger}$ Nano and Heterogeneous Materials Center, School of Materials Science and Engineering, Nanjing University of Science and Technology, Nanjing 210094, Jiangsu, China

Corresponding Author. Email: *weiliu@ njust.edu.cn 
Table S1. Adsorption energies of hexachlorobenzene at the high-symmetric sites of the $\operatorname{Pt}(111)$ with a $(3 \times 3)$ unit cell.

\begin{tabular}{ccc}
\hline \multirow{2}{*}{ Site } & \multicolumn{2}{c}{$E_{\text {ads }}(\mathrm{eV})$} \\
\cline { 2 - 3 } & physisorption & chemisorption \\
\hline bri0 & -0.97 & bri30 \\
bri30 & -0.98 & -0.94 \\
atop0 & -0.67 & $/$ \\
atop30 & -0.92 & $/$ \\
fcc $0^{\circ}$ & -0.71 & bri30 \\
fcc30 $30^{\circ}$ & -0.94 & bri30 \\
hcp0 $0^{\circ}$ & $/$ & bri30 \\
hcp30 & -0.93 & bri30 \\
\hline
\end{tabular}


Figure S1. Projected density of states (PDOS) on the physisorbed molecules at high-symmetric sites.

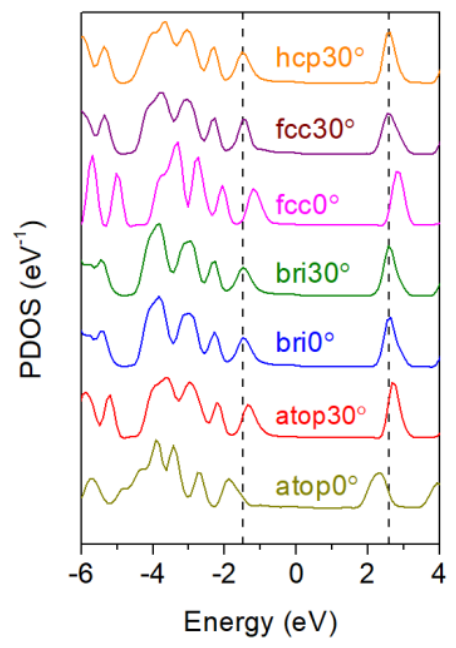


Figure S2. Projected density of states (PDOS) on $d$ band of the surface atoms at (a) $0.11 \mathrm{ML}$, (b) $0.06 \mathrm{ML}$, (c) $0.04 \mathrm{ML}$, and (d) $0 \mathrm{ML}$. The vertical red lines present the $d$ band center of the surface atoms.

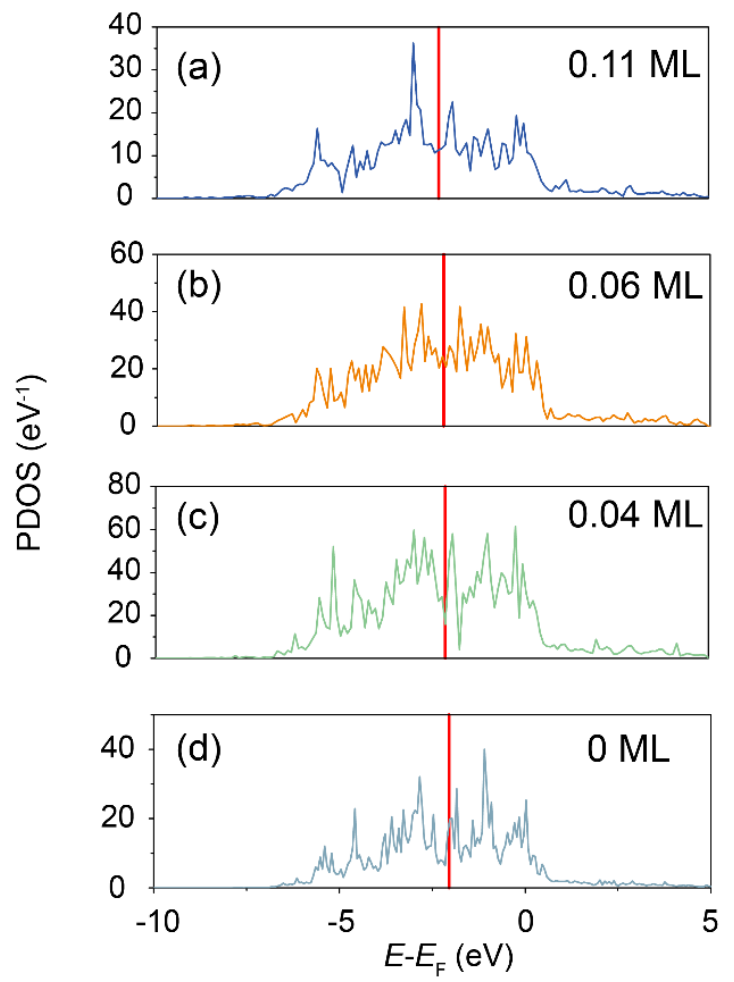

Table S2. $d$-band center of surface atoms with the chemisorbed $\mathrm{C}_{6} \mathrm{Cl}_{6}$.

\begin{tabular}{ccccc}
\hline & $0.11 \mathrm{ML}$ & $0.06 \mathrm{ML}$ & $0.04 \mathrm{ML}$ & $0 \mathrm{ML}$ \\
\hline $\begin{array}{c}d \text {-band center } \\
(\mathrm{eV})\end{array}$ & -2.34 & -2.23 & -2.14 & -2.05 \\
\hline
\end{tabular}


Figure S3. Halogen bond interactions diagram. (a) Anisotropic charge distribution of the halogen atom, where the electronics are accumulated around the waist and a $\sigma$-hole is induced on the head. (b) and (c) Two distinct pattern of R-X trimer. A high electronics depletion region would be induced in the pattern $\mathrm{b}$, which results in a low thermal stability. Therefore, the $\mathrm{R}-\mathrm{X}$ would rotate a slight angle to position the $\mathrm{R}-\mathrm{X} \cdots \mathrm{X}$ on a straight line. The rotation angle is related on the bond length of $\mathrm{R}-\mathrm{X}$ and interatomic distance of halogen.

(a)

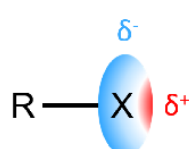

(b)

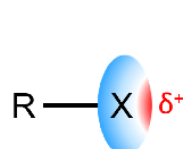

(c)

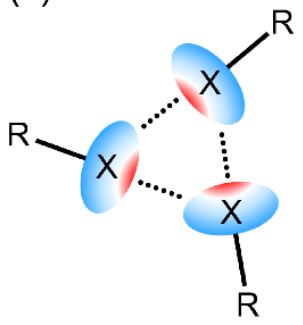


Figure S4. Simulated scanning tunneling microscope (STM) image. (a) and (c) STM imagine of hexachlorobenzene at the physisorbed and chemisorbed states on the $\mathrm{Pt}(111)$ with a $(4 \times 4)$ unit cell, respectively. (b) and (d) STM imagine of hexachlorobenzene at the physisorbed and chemisorbed states on the $\mathrm{Pt}(111)$ with a $(5 \times 5)$ unit cell, respectively.
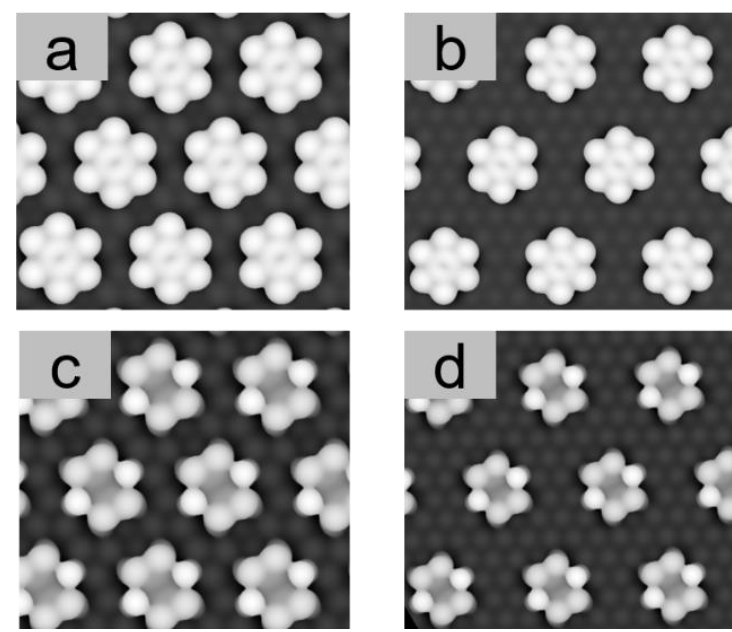
Figure S5. Potential energy curve of the hexachlorobenzene on the $\operatorname{Pt}(111)$ surface at different coverage. Adsorption energy of hexachlorobenzene using (a) a ( $3 \times 3)$ unit cell, (b) a (4×4) unit cell, (c) a $(5 \times 5)$ unit cell.

(a)

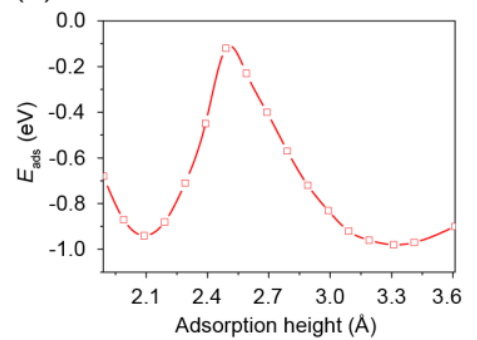

(b)

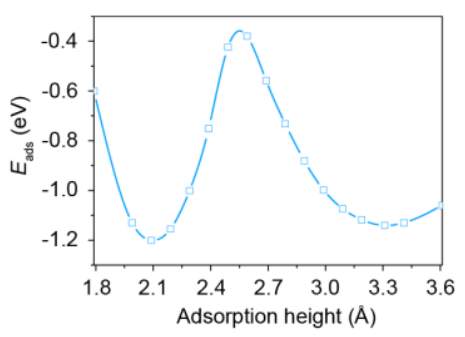

(c)

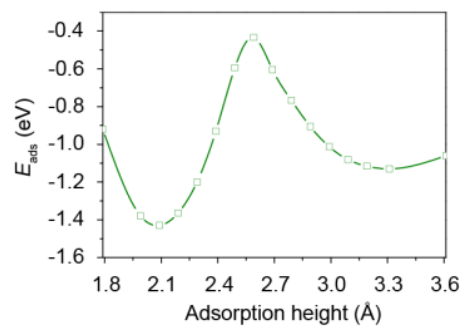

Table S3. Adsorption energies obtained with $\mathrm{PBE}+\mathrm{vdW}^{\text {surf }} E_{\text {ads }}(\mathrm{eV})$, adsorption heights of $\mathrm{C}$ atoms $h_{1}(\AA)$ and $\mathrm{Cl}$ atoms $h_{2}(\AA)$ for hexachlorobenzene on $\operatorname{Pt}(111)$ at different coverages (ML).

\begin{tabular}{cccccc}
\hline supercell & coverage & states & $E_{\text {ads }}$ & $h_{1}$ & $h_{2}$ \\
\hline \multirow{2}{*}{$(3 \times 3)$} & \multirow{2}{*}{0.1111} & on & -0.94 & 2.08 & $2.77 / 3.38$ \\
& & off & -0.98 & 3.31 & 3.33 \\
\multirow{2}{*}{$(4 \times 4)$} & \multirow{2}{*}{0.0625} & on & -1.20 & 2.11 & $2.80 / 3.29$ \\
& & off & -1.14 & 3.26 & 3.27 \\
$(5 \times 5)$ & \multirow{2}{*}{0.04} & on & -1.43 & 2.10 & $2.78 / 3.31$ \\
& & off & -1.13 & 3.23 & 3.23 \\
\hline
\end{tabular}


Figure S6. (a) Projected density of states (PDOS) of physisorbed molecules onto the atomic orbitals $\left(s, p_{x}, p_{y}\right.$, and $p_{z}$ ). (b) Zoom-in imagine of PDOS near the fermi level.

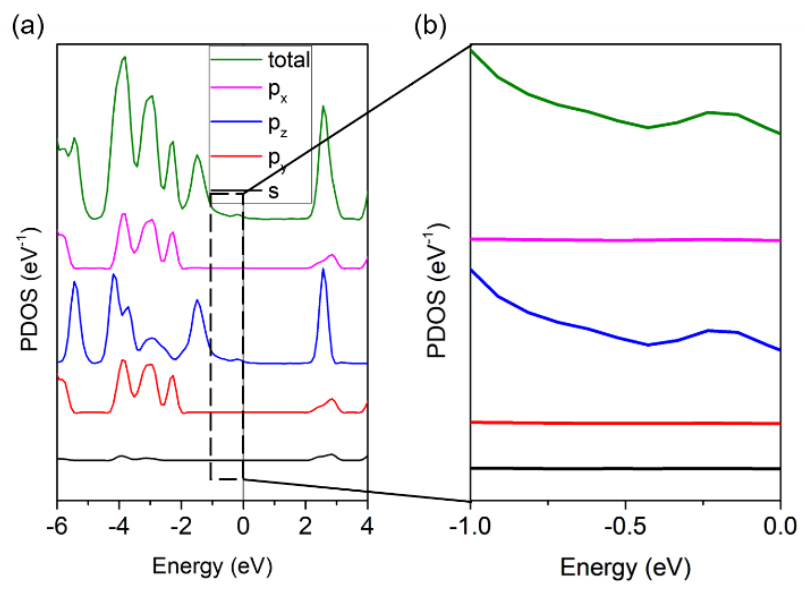


Figure S7. Molecular orbital density of states (MODOS). (a) MODOS of hexachlorobenzene at the physisorbed (upper) and chemisorbed (lower) states on the $\mathrm{Pt}(111)$ surface with a $(4 \times 4)$ unit cell. (b) MODOS of hexachlorobenzene at the physisorbed (upper) and chemisorbed (lower) states on the $\operatorname{Pt}(111)$ surface with a $(5 \times 5)$ unit cell.

(a)

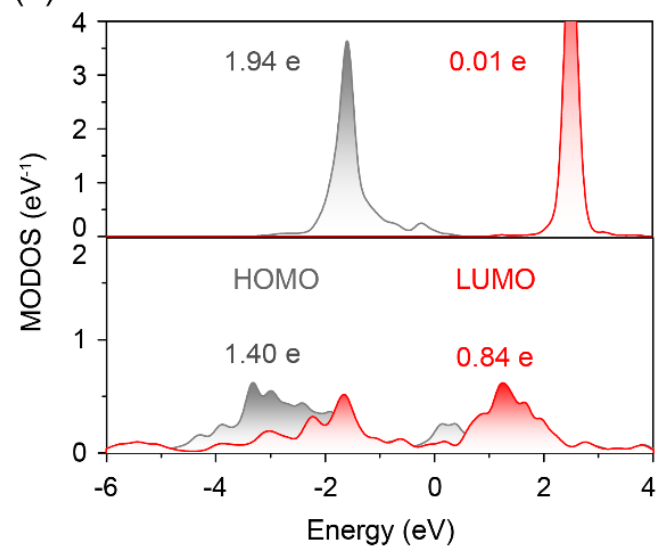

(b)

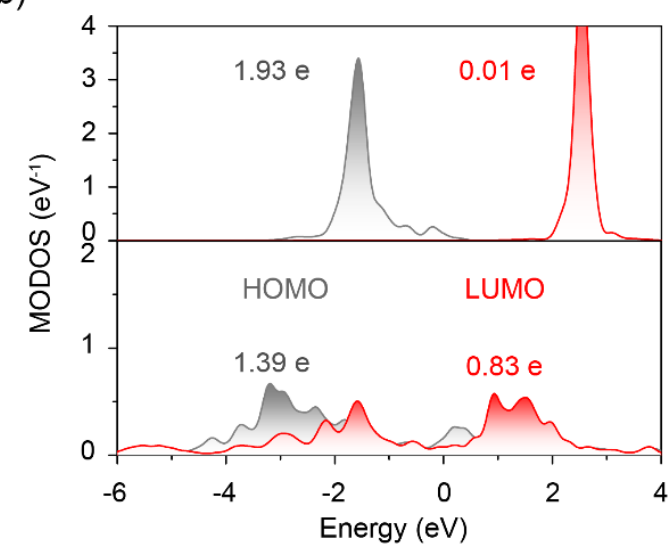


Figure S8. Transmission pathway of the integrated circuit. (a) and (b) Electrode model and transmission pathway when the molecule at the physisorbed and chemisorbed states, respectively. Zoom-in images show the details at the interface.

(a)

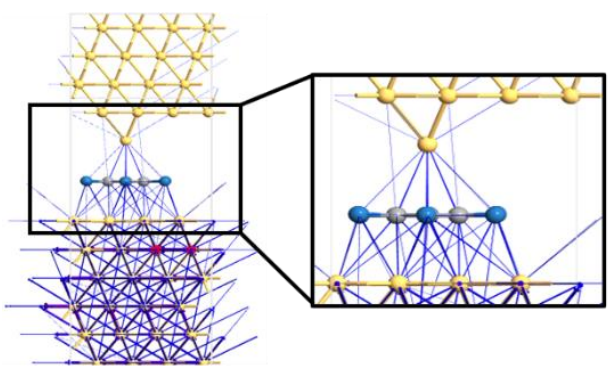

(b)

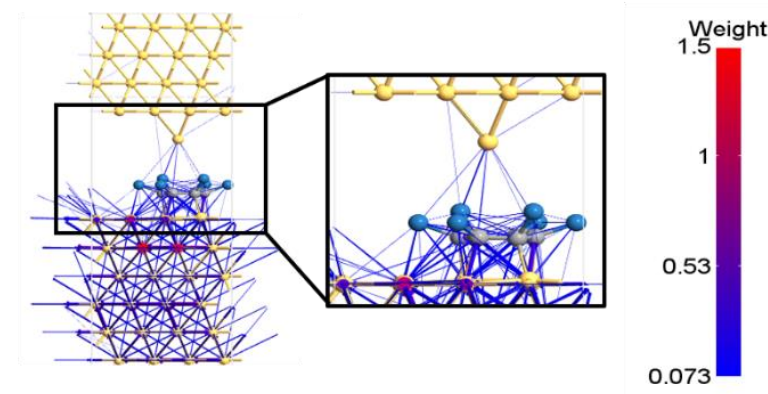

